\title{
EVALUACIÓN DE UN PROGRAMA DE INTERVENCIÓN PARA PROMOVER LA SALUD SEXUAL EN ADOLESCENTES MEXICANOS EN CONFLICTO CON LA LEY
}

\author{
Ricardo Sánchez Medina*, David Javier Enríquez Negrete y Silvia Susana Robles Montijo \\ Facultad de Estudios Superiores Iztacala, Universidad Nacional Autónoma de México.
}

Recibido, septiembre $3 / 2015$

Concepto evaluación, mayo 17/2016

Aceptado, junio 8/2016

\author{
Referencia: Sánchez Medina, R., Enríquez Negrete, D. J. \& Robles \\ Montijo, S. S. (2016). Evaluación de un programa de intervención \\ para promover la salud sexual en adolescentes mexicanos en con- \\ flicto con la ley. Acta Colombiana de Psicología, 19(2), 257-268. \\ DOI: 10.14718/ACP.2016.19.2.11
}

Resumen

El propósito de la presente investigación fue evaluar la efectividad de un programa de intervención para promover la salud sexual de adolescentes que se encuentran en conflicto con la ley. Se trabajó con 118 adolescentes pertenecientes a una comunidad de diagnóstico. Se utilizó un diseño cuasiexperimental con un grupo de intervención y uno control, conformado por tres fases: preevaluación, intervención y postevaluación. La intervención consistió en cinco sesiones de dos horas cada una, y estuvo conformada por los elementos que responden al modelo ecológico en sus tres niveles: microsistema (conocimientos, creencias, actitudes, intención y autoeficacia para usar condón, autoestima, comunicación con pareja, estilos de negociación para usar condón, entre otras); mesosistema (apoyo, supervisión y comunicación con padre y madre, entre otras), y macrosistema (estereotipos de género). Se realizó un análisis de medidas repetidas. Los resultados mostraron cambios entre el grupo control y el experimental, a favor de este último en todas las variables del modelo, excepto en comunicación con la pareja y autoestima. Los hallazgos se discuten en términos de la importancia de diseñar programas con base en el modelo ecológico dirigidos a población que se encuentran en un ambiente privado de la libertad.

Palabras clave: modelo ecológico, adolescentes, uso de condón, intervención, VIH/Sida.

\section{EVALUATION OF AN INTERVENTION PROGRAM TO PROMOTE SEXUAL HEALTH AMONG MEXICAN ADOLESCENTS IN CONFLICT WITH THE LAW}

Abstract

The purpose of this research was to evaluate the effectiveness of an intervention program to promote sexual health of adolescents in conflict with the law. The study was carried out with 118 adolescents belonging to a diagnosis community. A quasi-experimental design was used with an intervention group and one control group, consisting of three phases: preevaluation, intervention and post-evaluation. The intervention, which consisted of five sessions of two hours each one, comprised the elements that respond to the ecological model in its three levels: micro-system (knowledge, believes, attitudes, intentions and self-efficacy to use condoms, self-esteem, communication with partner, negotiating styles, etc.); meso-system (support, supervision and communication with parent, etc.) and macro-system (gender stereotypes). An analysis of repeated measures was performed. Results showed changes between control and experimental groups, in favor of the latter in all the variables of the model, except for partner communication and self-esteem. The findings are discussed in terms of the importance of designing programs based on the ecological model aimed at people who are deprived of freedom.

Key words: ecological model, adolescents, condom use, intervention, HIV/AIDS.

\footnotetext{
* Av. De los Barrios № 1, Los Reyes Iztacala, Tlalnepantla, Edo. de México, México. Torre Académica, segundo piso, cubículo 14. Tel.+52 (55) 56231333 ext.39855.ricardo.sanchez@ired.unam.mx
} 


\title{
AVALIAÇÃO DE UM PROGRAMA DE INTERVENÇÃO PARA PROMOVER A SAÚDE SEXUAL DE ADOLESCENTES MEXICANOS EM CONFLITO COM A LEI
}

\author{
Resumo
}

\begin{abstract}
O propósito desta pesquisa foi avaliar a efetividade de um programa de intervenção para promover a saúde sexual de adolescentes que se encontram em conflito com a lei. Trabalhou-se com 118 adolescentes pertencentes a uma comunidade de diagnóstico. Foi utilizado um desenho quase-experimental com um grupo de intervenção e um de controle, conformado por três fases: pré-avaliação, intervenção e pós-avaliação. A intervenção consistiu em cinco sessões de duas horas cada uma e foi formada pelos elementos que respondem ao modelo ecológico em seus três níveis: microssistema (conhecimentos, crenças, atitudes, intenção e autoeficácia para usar preservativo, autoestima, comunicação com o parceiro, estilos de negociação para usar preservativo, entre outros); mesossistema (apoio, supervisão e comunicação com os pais, entre outros) e macrossistema (estereótipos de gênero). Foi realizada uma análise de medidas repetidas. Os resultados mostraram mudanças entre o grupo controle e o experimental a favor deste último em todas as variáveis do modelo, exceto em comunicação com o parceiro e autoestima. Os achados são discutidos quanto à importância de desenhar programas com base no modelo ecológico dirigidos à população que se encontra num ambiente privado de liberdade.

Palavras-chave: modelo ecológico, adolescentes, uso de preservativo, intervenção, HIV/Aids.
\end{abstract}

\section{INTRODUCCIÓN}

Instituciones a nivel internacional han creado diversos programas para disminuir los altos índices de casos de personas infectadas por el virus de la inmunodeficiencia humana (VIH), ya que hasta el momento se han reportado 36,9 millones de personas diagnosticadas con el virus (Programa Conjunto de las Naciones Unidas sobre el VIH/ SIDA [ONUSIDA], 2016). Una de las formas que más se ha difundido para prevenirlo es la utilización correcta y consistente del condón; sin embargo, a pesar de que existe información sobre cómo usarlo y por qué se debería usar, las personas no lo utilizan en sus relaciones sexuales, lo cual propicia el riesgo de contraer VIH/SIDA(ONUSIDA, 2016).

ONUSIDA(2014) señala que un entorno social como el carcelario se relaciona con determinados comportamientos que hacen más probable la presencia de problemas de salud sexual. El Ministerio de la Protección Social de Colombia (2011) menciona que las prisiones en Latinoamérica son ambientes altamente vulnerables a la infección por VIH, dado que la prevalencia en estos sitios puede llegar a ser hasta veinte veces más alta que en la población general; y además, son lugares más susceptibles a presentar violencia con armas cortopunzantes, maltrato y discriminación, así como abuso sexual y ejercicio de distintas formas de violencia física y psicológica. En estos entornos, los adolescentes, en particular presentan mayor frecuencia a la exposición a factores de riesgo, como el consumo de drogas, aumento de conductas delictivas, desintegración familiar y mayor insatisfacción social (Andrade, Barbosa y Lozada, 2012; Sanabria y Uribe, 2010).

De acuerdo con ONUSIDA (2009), los programas de prevención, tratamiento, atención y apoyo contra el VIH no se encuentran en un nivel de desarrollo y ejecución adecuados para responder al problema en las cárceles. En estos entornos, en general, no se abordan las neceSIDAdes específicas de cada género, ni tampoco se toma en consideración que el comportamiento sexual de las personas encarceladas puede tener un impacto no solo en ellas mismas, sino en sus parejas sexuales, sus familias y las comunidades en las que viven. Por esto, que se ha sugerido que las intervenciones deben estar centradas en brindar información sobre las vías de transmisión del VIH y las formas de prevenirlo, clarificar mitos con respecto a sus formas de transmisión, promover entre los participantes el uso del condón y reducir el daño entre usuarios de drogas inyectadas (Senteio, Wright, Jackson, Welk y Zhang, 2010; Thurman, Kidman, Carton y Chiroro, 2016).

A pesar de que hay directrices sobre las variables en las cuales se debe intervenir, trabajar en la prevención del VIH/SIDA es sumamente complejo por la gran cantidad de factores que se encuentran entremezclados (DiClemente, Salazar y Crosby, 2007); por ejemplo, algunos programas consideran que es necesario proporcionar información y modificar algunos aspectos psicosociales, como las creencias, intenciones y actitudes, entre otras (Operario, Smith, Arnold y Kegeles, 2010); sin embargo, tales acciones por sí solas no son suficientes ya que a pesar de que se logren cambios en estas variables, estas no necesariamente se vinculan con el comportamiento sexual, como, por ejemplo, en el uso del condón.

En ese sentido, se requiere el desarrollo de habilidades conductuales que coadyuven a tener un comportamiento sexual seguro (Zimmerman et al., 2008), considerando necesariamente que las personas se encuentran en un contexto social determinado, y que no solo se requiere que adquieran ciertos repertorios, sino que también sean conscientes de su contexto inmediato para analizar qué apoyo pueden o 
no recibir de su medio social, y que puedan saber cuáles podrían ser las consecuencias de su proceder.

Teniendo en cuenta lo anterior, es relevante tener en cuenta la propuesta del modelo ecológico de Bronfenbrenner (1987), la cual permite analizar al individuo en relación con los diferentes contextos en los que se encuentra inmerso. Desde este modelo, en el nivel micro (microsistema) se contemplan aquellas variables como conocimientos, actitudes, intenciones y autoeficacia, entre otras, relacionadas con el comportamiento sexual; en el nivel meso, o intermedio (mesosistema), se encuentran el apoyo, la supervisión familiar y la norma subjetiva del grupo de pares; $y$ en el nivel macro (macrosistema) aparecen los factores socioeconómicos, ideológicos y culturales en los que se desenvuelve la persona, como, por ejemplo, los estereotipos de género (DiClemente, Salazar y Crosby, 2007).

Las investigaciones que parten del modelo ecológico en su mayoría han sido de corte teórico y hablan de la importancia de intervenir para producir cambios en todas las variables del modelo (Trickett, 2009); por otro lado, entre los escasos estudios de corte empírico, solo se retoman dos niveles del modelo, ya sea el micro y el meso, o el micro y el macro, los cuales, por ejemplo, en la investigación de Pinto y McKay, (2006), únicamente evalúan las habilidades de los adolescentes y su relación con la comunicación y supervisión parental, pero no abordan los tres niveles en su conjunto; e incluso, tan solo dan elementos por considerar en los programas de intervención, pero no los llevan a cabo. En este sentido, surge la neceSIDAd de generar programas de intervención, considerando cada una de las variables de los diferentes sistemas y, además, evaluar su efectividad.

Tomando en consideración el modelo ecológico, el propósito del presente estudio fue evaluar los efectos de un programa de intervención, que incluye distintas variables definidas en el micro, meso y macrosistema, en una muestra de varones adolescentes que se encuentran en conflicto con la ley.

Específicamente, esta investigación se realizó en una de las seis Comunidades Especializadas de Atención para Adolescentes, que dependen de la Dirección General de Tratamiento para Adolescentes (DGTPA) de la Subsecretaría de Sistema Penitenciario del Gobierno del Distrito Federal en la Ciudad de México (DGTPA, 2013).

\section{MÉTODO}

\section{Diseño}

Para el estudio se utilizó un diseño pretest-postest con un grupo experimental y uno control (Kerlinger y Lee, 2002) que se llevó a cabo en tres fases: preevaluación, intervención y postevaluación; por lo que ambos grupos fueron evaluados antes y después de la intervención. Solo el grupo experimental recibió el programa de intervención, mientras que el grupo control recibió el entrenamiento del uso correcto del condón e información sobre la prevención del VIH/SIDA una vez terminadas las evaluaciones.

\section{Participantes}

El estudio se llevó a cabo con 118 varones pertenecientes a una comunidad de diagnóstico de la ciudad de México, la cual estaba conformada por ocho dormitorios, donde, en promedio, en cada uno convivían entre 25 y 35 adolescentes. A través de un muestreo por conglomerados (Kerlinger y Lee, 2002), se eligieron cuatro dormitorios al azar, dos de estos se asignaron aleatoriamente al grupo control $(n=50)$ y los otros dos al grupo experimental $(n=68)$. Con el propósito de saber si los grupos eran homogéneos en términos de sus características sociodemográficas, se llevó a cabo un análisis de diferencias entre grupos, y no se encontraron diferencias significativas. La edad promedio de los adolescentes fue de 16 años, la mayoría de ellos eran solteros, tenían estudios de primaria y secundaria, vivían con sus padres, sus ingresos mensuales eran de menos de 4.000 pesos mexicanos, y el delito por el cual había ingresado la mayoría fue el robo.

\section{Variables}

Variables dependientes. De acuerdo con el modelo ecológico de Bronfenbrenner (1987) y la propuesta aplicada a la prevención del VIH/SIDA de DiClemente, Salazar y Crosby (2007), las variables del microsistema son: (1) conocimientos sobre VIH/SIDA-ITS; (2) conocimientos sobre el uso de condón; (3) creencias y actitudes hacia el uso del condón; (4) intención y autoeficacia para usar condón en relaciones sexuales; (5) autoeficacia para usar condón en situaciones de riesgo;(6) autoestima; (7) comunicación sobre temas sexuales con la pareja; (8) estilos de negociación con respecto al uso del condón; (9) autoeficacia para iniciar una conversación sobre temas sexuales con padre, madre y pareja; (10) autoeficacia para evitar conductas de riesgo; (11) habilidad para usar un condón en situaciones simuladas, y (12) habilidad para negociar el uso del condón. Por otro lado, las variables del mesosistema son: (1) apoyo paterno y materno; (2) supervisión paterna y materna; (3) comunicación sexual entre padres e hijos, y (4) norma subjetiva del grupo de pares. Y, finalmente, la variable del macrosistema es estereotipos de género.

Variable independiente. Se consideró como variable independiente el programa de intervención conformado por los siguientes elementos que, de igual forma, responden al modelo ecológico: 
Para el microsistema, el programa incluye: a) información, análisis y discusión sobre la prevención, transmisión y sintomatología del VIH/SIDA y las ITS, y sobre la prevención de embarazos no planeados; b) ventajas y desventajas del uso del condón en relaciones sexuales; c) comunicación efectiva y su importancia en la relación de pareja; d) el papel de la autoestima en una relación de pareja saludable; y e) análisis y discusión grupal sobre comportamientos sexuales de riesgo y situaciones en las que se presentan, enfatizando el papel que tiene el uso de drogas o alcohol en el desarrollo de conductas sexuales preventivas.

Para el mesosistema incluye: a) análisis y discusión grupal sobre la supervisión parental y apoyo familiar sobre el comportamiento sexual de los hijos; y b) ventajas y desventajas de la presión del grupo de pares sobre el comportamiento sexual.

Y para el macrosistema: a) análisis y discusión sobre las diferencias entre hombres y mujeres en el terreno sexual; y b) análisis y discusión de las creencias familiares sobre la sexualidad y sobre los mitos de la homosexualidad.

\section{Instrumentos}

Se utilizó la Escala de Salud Sexual de Robles y DíazLoving (2011), la cual está compuesta por diversos instrumentos de evaluación que permiten evaluar variables que pertenecen al microsistema, para las variables del meso y del macro se buscaron instrumentos adicionales que tuvieran buenas propiedades psicométricas. La consistencia interna de los instrumentos fue evaluada a partir del alfa de Cronbach.

\section{Reporte de datos sociodemográficos}

Se diseñaron seis reactivos para obtener información de los adolescentes sobre su edad (años cumplidos), estado civil (soltero, casado, unión libre), nivel de estudios (ninguno, primaria, secundaria, preparatoria, univerSIDAd, posgrado, carrera técnica, otro), con quién vivían en el momento de su detención (con su padre, con su madre, con ambos padres, otros) e ingresos familiares (menos de $\$ 2000$, de $\$ 2001$ a $\$ 3999$, de $\$ 4000$ a $\$ 5999$, de $\$ 6000$ a $\$ 9999$, más de $\$ 10000$ o no sé). Asimismo, se obtuvo información sobre datos jurídicos referentes al delito por el cual fueron acusados (robo, extorsión, lesiones, homicidio, privación ilegal de la libertad, o delincuencia organizada).

\section{Prueba de conocimientos sobre VIH/SIDA-ITS-uso de condón}

Se utilizaron doce reactivos relacionados con conocimientos sobre el VIH/SIDA, la prevención, transmisión, sintomatología y características de ITS; y seis reactivos relacionados con conocimientos sobre el uso correcto del condón (Robles y Díaz-Loving, 2011); los cuales se presentaron en un formato de respuesta de cierto, falso y no sé. La prueba contó con un índice de dificultad de 0.52 , el cual se obtuvo a partir de dividir el número de participantes que respondieron incorrectamente, entre el total de participantes que respondieron al reactivo.

\section{Escala de creencias hacia el uso del condón}

Doce reactivos que evalúan creencias hacia el uso del condón con pareja regular (Robles y Díaz-Loving, 2011), presentados en una escala tipo Likert de 1 (totalmente en desacuerdo) a 5 (totalmente de acuerdo). Cuatro de estos se orientaron a creencias negativas y ocho a creencias de valoración propia. La consistencia interna total fue de $\alpha=0.84$.

\section{Escala de actitudes hacia el uso del condón}

Cinco reactivos con opciones de respuesta definidas en una escala de diferencial semántico de 1 a $7(\alpha=0.87)$, de Robles y Díaz-Loving (2011).

Intención para usar condón en relaciones sexuales

Un reactivo que evalúa la probabilidad de uso condón en su próxima relación sexual con pareja regular, en una escala de 7 puntos ( $1=$ nada probable a $7=$ totalmente probable $)$.

Autoeficacia para usar condón en relaciones sexuales

Un reactivo en una escala de 1 a 7 que evalúa qué tan seguros están de que puedan usar un condón cada vez que tienes sexo vaginal con su pareja estable, donde 1 es muy seguro de no poder usarlo y 7 muy seguro de poder usarlo.

Escala de autoeficacia para usar condón en situaciones de riesgo

Un reactivo que evalúa qué tan seguros están de poder usar siempre un condón durante el sexo vaginal en siete situaciones de riesgo (Robles y Díaz-Loving, 2011) con opciones de respuesta en una escala de 1 (muy seguro de no poder usarlo) a 7 (muy seguro de poder usarlo). La consistencia interna fue de $\alpha=0.78$.

\section{Escala de autoestima}

Se utilizaron diez reactivos para evaluar el grado de autoestima (Díaz, 2010), los cuales tuvieron un formato de respuesta en escala tipo Likert con valores de 1 (muy de acuerdo) a 4 (muy en desacuerdo); la consistencia interna de la escala fue de $\alpha=0.72$.

Escala de comunicación sobre temas sexuales con la pareja

Diez reactivos midieron la frecuencia con la cual los jóvenes se comunican con su pareja sobre temas sexuales 
(Robles y Díaz-Loving, 2011); las opciones de respuesta tuvieron valores de 1 (nunca) a 5 (siempre). Su consistencia interna fue de $\alpha=0.94$.

Inventario de estilos de negociación sobre el uso del condón.

Quince reactivos midieron los estilos colaboración y equidad (Robles y Díaz-Loving, 2011) que utilizan los participantes al negociar el uso del condón con su pareja; las opciones de respuesta tuvieron un formato en escala tipo Likert con valores de 1 (totalmente en desacuerdo) a 5 (totalmente de acuerdo). La consistencia interna fue de $\alpha=0.85$.

Autoeficacia para iniciar una conversación sobre temas sexuales con padre, madre y pareja

Se diseñaron tres reactivos para evaluar la capacidad de los adolescentes para iniciar una conversación con su padre, madre y pareja, cada uno con formato de respuesta en escala tipo Likert, cuyos valores fueron de 1 ( de no poder hacerlo) a 7 (muy seguro de poder hacerlo).

Escala de autoeficacia para evitar conductas de riesgo

Se retomó la escala de Gaxiola, González y Contreras (2011) y se adaptó la pregunta que hace referencia a lo que hacen sus amigos por qué tan seguro estás de poder hacer lo contrario a lo que hacen tus amigos cuando...; las opciones fueron ...roban y ...hacen daño a lugares públicos; el formato de respuesta estuvo en una escala de siete puntos, con valores de 1 (absolutamente seguro de que no puedo) a 7 (absolutamente seguro de que sí puedo).

Habilidad demostrada para usar un condón en situaciones simuladas

Esta variable hace referencia a la forma en la que se coloca y retira un preservativo en un modelo de pene para la enseñanza. A través de la observación directa se registró en cada participante la ocurrencia de 8 pasos para colocar y retirar un condón en un pene de enseñanza; para esto se siguió el procedimiento utilizado por Robles, Rodríguez, Frías y Moreno (2014).

Observación directa de la habilidad para negociar el uso del condón

La habilidad fue evaluada con base en la propuesta de Tulloch, McCaul, Miltenberger y Smyth (2004). Se realizó un juego de roles en el que el adolescente debía negociar con un confederado el uso del condón, y un observador previamente entrenado evaluó en cada participante sus estrategias (argumentos) utilizadas para negociar el condón con su pareja, asignando un máximo de cuatro puntos en la siguiente escala: $1=$ inapropiado/inefectivo, $2=$ menciona el uso del condón pero no provee argumentos para su uso, $3=$ da argumentos para usar el condón, pero no responde a los argumentos que da la pareja para no usarlo, y $4=$ responde efectivamente a los argumentos que da la pareja para no usar el condón y da argumentos de por qué usarlo.

\section{Escala de apoyo paterno y materno}

Ocho reactivos evaluaron la percepción de los hijos sobre el apoyo recibido por parte de sus padres y de sus madres en un periodo de tres meses (Sánchez, Enríquez y Reyes-Lagunes, 2011). Los reactivos tuvieron un formato de respuesta en escala tipo Likert con valores de 1(nada) a 5 (mucho). La consistencia interna para apoyo paterno fue $\alpha=0.98$ y para apoyo materno $\alpha=0.97$.

\section{Escala de supervisión paterna y materna}

Diez reactivos evaluaron la percepción de los participantes sobre la supervisión recibida por parte de sus padres y de sus madres en un periodo de tres meses (Sánchez, Enríquez y Reyes-Lagunes, 2011). Los reactivos tuvieron un formato de respuesta en escala tipo Likert con valores de 1 (nada) a 5 (mucho). La consistencia interna para supervisión paterna fue $\alpha=0.92$ y para supervisión materna $\alpha=0.95$.

Escala de comunicación sexual entre padres e hijos

Diez reactivos midieron la frecuencia con la cual los jóvenes se comunican con su padre $(\alpha=0.96)$ y madre $(\alpha=0.98)$ sobre temas sexuales (Robles y Díaz-Loving, 2011); las opciones de respuesta tuvieron valores de 1 (nunca) a 5 (siempre).

\section{Norma subjetiva del grupo de pares}

Un reactivo midió lo que los jóvenes creen que piensan sus amigos sobre usar condón con pareja regular; las opciones de respuesta tuvieron valores de 1 (mis amigos piensan que no debería usarlo) a 7 (mis amigos piensan que sí debería usarlo).

\section{Escala de estereotipos de género}

Trece reactivos midieron la visión estereotipada que los jóvenes tienen sobre hombres y mujeres en el ámbito interpersonal y en el contexto social (Rocha y Díaz-Loving, 2005); las opciones de respuesta estuvieron definidas en una escala de cinco puntos evaluando el grado de acuerdo con cada afirmación. Se obtuvo una consistencia interna de $\alpha=0.89$.

\section{Procedimiento}

Inicialmente, se les solicitó consentimiento informado a los padres antes de hacer cualquier evaluación a los adoles- 
centes de las habitaciones seleccionadas de la comunidad de diagnóstico; a los hijos cuyos padres aceptaron su participación se les entregó el consentimiento informado, haciendo hincapié en que la información sería confidencial y que la institución no tendría acceso a la información individual.

Una vez firmados los consentimientos, se aplicó el conjunto de instrumentos de evaluación (pretest) a los adolescentes de los dos grupos en un salón ubicado en el área de visita familiar donde se colocaron sillas en círculo y habían tres mesas disponibles para el trabajo en equipo. Se evaluó a cada participante por separado, pero la habilidad para usar correctamente el condón en un modelo de pene para enseñanza, y la habilidad para negociar el uso del condón con su pareja se evaluaron a partir de un juego de roles.

Una semana después, el grupo experimental recibió el programa de intervención que constó de cinco sesiones (una por semana) de dos horas cada una y que consistió en la ejecución de las siguientes actividades:

Sesión 1: Dinámica sobre la transmisión del VIH/SIDA; y dinámica sobre las ventajas y desventajas de usar condón en las relaciones sexuales.

Sesión 2: Discusión de diversas situaciones problemáticas, donde se debatían las implicaciones de cada situación y la utilización del uso del condón; y dinámica sobre mitos hacia la homosexualidad y sobre lo que implica ser hombre y ser mujer en el terreno sexual.

Sesión 3: Discusión sobre la importancia de la familia en el comportamiento sexual; y entrenamiento en la habilidad de comunicación sexual con los padres a través de cuatro técnicas de cambio conductual que constituyen el aprendizaje estructurado: modelamiento, juego de roles, retroalimentación y entrenamiento para la transferencia.

Sesión 4: Dinámica del anuncio publicitario para promover el uso del condón; y entrenamiento en habilidades del uso correcto del condón utilizando un modelo de pene para la enseñanza.

Sesión 5: Entrenamiento en habilidades centrado en la negociación del uso del condón con base en las técnicas del aprendizaje estructurado.

\section{Análisis de datos}

Se empleó el paquete estadístico SPSS 20 para el análisis de datos. Se realizaron análisis de diferencias entre grupos para identificar la homogeneidad entre los grupos utilizando ji cuadrado para variables nominales y t de student para variables de intervalo. Para evaluar los efectos de la intervención entre ambos grupos se realizaron análisis de medidas repetidas, se consideraron los efectos principales del grupo, el efecto del tipo de tratamiento, y la interacción entre variables diferenciadas para cada grupo.

\section{RESULTADOS}

Los resultados que se presentan a continuación se encuentran divididos por sistemas, en función de la propuesta del modelo ecológico (Bronfenbrenner, 1987); en cada uno de ellos se analizan las diferencias entre grupos en función de los efectos de la intervención.

\section{Variables del microsistema}

En los resultados de la evaluación de conocimientos sobre VIH/SIDA-ITS-uso del condón antes y después de la intervención (véase Tabla 1) se observa que, en general, el grupo experimental obtuvo mayores porcentajes de respuestas correctas que el control en la postevaluación y que el efecto de interacción tuvo diferencias significativas entre los grupos.

En las comparaciones entre el grupo experimental y control sobre las variables psicosociales relacionadas con el uso del condón (véase Tabla 2) se encontraron diferencias significativas entre el puntaje obtenido entre pre y post diferenciado para cada grupo: en el grupo experimental es menor la puntuación de creencias negativas y mayor la puntuación en actitudes, intención y autoeficacia; en cambio, en el grupo control no se presentaron diferencias significativas. Respecto de las creencias de seguridad y protección para usar condón, el grupo experimental obtuvo ligeramente un mayor puntaje que el control; sin embargo, al analizar el efecto del tipo de tratamiento no se encontraron diferencias significativas entre la medición pre y post ni tampoco hubo interacción entre estas variables.

Con respecto a la variable autoestima, al revisar los efectos principales del grupo no se encontraron diferencias significativas $(\mathrm{F}=0.316, p=0.576)$; tampoco hubo efecto del tipo de tratamiento $(\mathrm{F}=2.98, p=0.089)$ ni efecto de interacción $(\mathrm{F}=0.271, p=0.605)$. En general, antes y después de la intervención los adolescentes reportaron tener una autoestima moderada; lo que indica que para esta variable la intervención realizada no tuvo efecto.

Al evaluar qué tan capaces se sienten los adolescentes de poder iniciar una conversación sobre temas sexuales con sus padres y su pareja (véase Tabla 3), se encontró que el grupo experimental obtuvo mayor puntaje en la autoeficacia, en comparación con el control; este último mantiene su nivel de autoeficacia de pre a post evaluación.

En los resultados correspondientes a la frecuencia de la comunicación con la pareja sobre temas sexuales y el estilo de negociación sobre el uso del condón (véase Tabla 4), en promedio, el grupo experimental obtuvo mayor puntaje que el de control; mientras que en la interacción de estas variables se encontró que existe un cambio en la puntuación entre pre y post diferenciado para cada grupo. 
Tabla 1.

Comparación entre los grupos experimental y control en la prueba de conocimientos sobre VIH/SIDA-ITS-uso de condón

\begin{tabular}{|c|c|c|c|c|c|c|c|}
\hline \multirow{2}{*}{ Variable } & \multicolumn{2}{|c|}{ Control } & \multicolumn{2}{|c|}{ Experimental } & \multicolumn{3}{|c|}{ ANOVA de medidas repetidas } \\
\hline & Pre & Post & Pre & Post & Grupo & Tratamiento & Interacción \\
\hline \multicolumn{8}{|c|}{ Conocimientos sobre VIH/SIDA } \\
\hline Sintomatología & $57.33 \%$ & $52.87 \%$ & $64.71 \%$ & $97.24 \%$ & $\begin{array}{l}\mathrm{F}=28.8 \\
\mathrm{p}<0.001\end{array}$ & $\begin{array}{l}F=7.79 \\
p<0.01\end{array}$ & $\begin{array}{c}\mathrm{F}=12.43 \\
\mathrm{p}<0.01\end{array}$ \\
\hline Transmisión & $39.33 \%$ & $35.63 \%$ & $42.65 \%$ & $94.44 \%$ & $\begin{array}{l}F=52.6 \\
p<0.001\end{array}$ & $\begin{array}{l}F=38.8 \\
p<0.001\end{array}$ & $\begin{array}{l}\mathrm{F}=45.9 \\
\mathrm{p}<0.001\end{array}$ \\
\hline Prevención & $44.67 \%$ & $39.08 \%$ & $50.00 \%$ & $97.22 \%$ & $\begin{array}{l}F=55.6 \\
p<0.001\end{array}$ & $\begin{array}{l}F=19.6 \\
p<0.001\end{array}$ & $\begin{array}{l}\mathrm{F}=29.0 \\
\mathrm{p}<0.001\end{array}$ \\
\hline ITS & $48.67 \%$ & $35.63 \%$ & $51.96 \%$ & $97.22 \%$ & $\begin{array}{l}F=47.4 \\
p<0.001\end{array}$ & $\begin{array}{l}F=19.0 \\
p<0.001\end{array}$ & $\begin{array}{l}\mathrm{F}=39.6 \\
\mathrm{p}<0.001\end{array}$ \\
\hline Global & $47.50 \%$ & $40.80 \%$ & $52.33 \%$ & $96.06 \%$ & $\begin{array}{l}\mathrm{F}=88.1 \\
\mathrm{p}<0.001\end{array}$ & $\begin{array}{l}F=30.5 \\
p<0.001\end{array}$ & $\begin{array}{l}\mathrm{F}=46.7 \\
\mathrm{p}<0.001\end{array}$ \\
\hline Uso de condón & $38.63 \%$ & $35.63 \%$ & $37.75 \%$ & $93.98 \%$ & $\begin{array}{l}F=66.1, \\
p<0.001\end{array}$ & $\begin{array}{l}\mathrm{F}=39.7 \\
\mathrm{p}<0.001\end{array}$ & $\begin{array}{l}\mathrm{F}=51.2 \\
\mathrm{p}<0.001\end{array}$ \\
\hline
\end{tabular}

Tabla 2.

Comparación entre los grupos experimental y control sobre las variables psicosociales sobre el uso del condón con pareja regular

\begin{tabular}{|c|c|c|c|c|c|c|c|}
\hline \multirow{2}{*}{ Variable } & \multicolumn{2}{|c|}{ Control } & \multicolumn{2}{|c|}{ Experimental } & \multicolumn{3}{|c|}{ ANOVA de medidas repetidas } \\
\hline & Pre & Post & Pre & Post & Grupo & Tratamiento & Interacción \\
\hline Creencias de seguridad y protección & $\begin{array}{c}3.66 \\
(1.49)\end{array}$ & $\begin{array}{c}3.34 \\
(1.58)\end{array}$ & $\begin{array}{c}4.18 \\
(1.06)\end{array}$ & $\begin{array}{l}4.75 \\
(.77)\end{array}$ & $\begin{array}{l}F=18.8, \\
p<0.001\end{array}$ & $\begin{array}{l}F=3.91, \\
p<0.052\end{array}$ & $\begin{array}{l}\mathrm{F}=1.51, \\
\mathrm{p}=0.223\end{array}$ \\
\hline Creencias negativas & $\begin{array}{c}3.70 \\
(1.26)\end{array}$ & $\begin{array}{c}3.34 \\
(1.37)\end{array}$ & $\begin{array}{c}3.22 \\
(1.11)\end{array}$ & $\begin{array}{l}2.81 \\
(.62)\end{array}$ & $\begin{array}{l}\mathrm{F}=5.85 \\
\mathrm{p}<0.05\end{array}$ & $\begin{array}{l}\mathrm{F}=9.99 \\
\mathrm{p}<0.01\end{array}$ & $\begin{array}{l}F=29.7 \\
p<0.001\end{array}$ \\
\hline Actitudes & $\begin{array}{c}3.34 \\
(1.95)\end{array}$ & $\begin{array}{c}3.52 \\
(2.26)\end{array}$ & $\begin{array}{c}3.58 \\
(2.05)\end{array}$ & $\begin{array}{c}6.64 \\
(1.19)\end{array}$ & $\begin{array}{l}\mathrm{F}=24.7 \\
\mathrm{p}<0.001\end{array}$ & $\begin{array}{l}F=26.7 \\
p<0.001\end{array}$ & $\begin{array}{l}\mathrm{F}=20.3 \\
\mathrm{p}<0.001\end{array}$ \\
\hline Intención para usarlo & $\begin{array}{l}3.76 \\
(2.63)\end{array}$ & $\begin{array}{c}3.55 \\
(2.50)\end{array}$ & $\begin{array}{c}4.29 \\
(2.59)\end{array}$ & $\begin{array}{c}6.36 \\
(1.71)\end{array}$ & $\begin{array}{l}F=16.8 \\
p<0.001\end{array}$ & $\begin{array}{l}F=2.54 \\
p<0.116\end{array}$ & $\begin{array}{l}\mathrm{F}=6.32 \\
\mathrm{p}<0.05\end{array}$ \\
\hline Autoeficacia para usarlo & $\begin{array}{c}3.52 \\
(2.58)\end{array}$ & $\begin{array}{c}3.24 \\
(2.53)\end{array}$ & $\begin{array}{c}4.32 \\
(2.66)\end{array}$ & $\begin{array}{c}6.78 \\
(1.01)\end{array}$ & $\begin{array}{l}\mathrm{F}=23.1 \\
\mathrm{p}<0.001\end{array}$ & $\begin{array}{l}F=9.56 \\
p<0.005\end{array}$ & $\begin{array}{l}\mathrm{F}=16.0 \\
\mathrm{p}<0.001\end{array}$ \\
\hline $\begin{array}{l}\text { Autoeficacia para usarlo en } \\
\text { situaciones de riesgo }\end{array}$ & $\begin{array}{l}3.18 \\
(2.11)\end{array}$ & $\begin{array}{c}2.97 \\
(2.17)\end{array}$ & $\begin{array}{l}3.35 \\
(2.19)\end{array}$ & $\begin{array}{c}6.50 \\
(1.54)\end{array}$ & $\begin{array}{l}\mathrm{F}=24.6 \\
\mathrm{p}<0.001\end{array}$ & $\begin{array}{l}\mathrm{F}=27.3 \\
\mathrm{p}<0.001\end{array}$ & $\begin{array}{l}\mathrm{F}=29.9 \\
\mathrm{p}<0.001\end{array}$ \\
\hline
\end{tabular}

Nota: En las medidas pre y post se muestra la puntuación obtenida y, entre paréntesis, la desviación estándar.

$\underline{\text { Tabla } 3}$

Comparación entre los grupos experimental y control sobre la autoeficacia para iniciar una conversación

\begin{tabular}{lccccccc}
\hline \multirow{2}{*}{ Variable } & \multicolumn{2}{c}{ Control } & \multicolumn{2}{c}{ Experimental } & \multicolumn{3}{c}{ ANOVA de medidas repetidas } \\
& Pre & Post & Pre & Post & Grupo & Tratamiento & Interacción \\
\hline \multirow{2}{*}{ Con padre } & 3.7 & 3.31 & 3.47 & 6.47 & $\mathrm{~F}=19.8$, & $\mathrm{F}=18.7$, & $\mathrm{F}=25.3$, \\
& $(2.12)$ & $(2.18)$ & $(2.10)$ & $(1.42)$ & $\mathrm{p}<0.001$ & $\mathrm{p}<0.001$ & $\mathrm{p}<0.001$ \\
Con madre & 4.56 & 4.38 & 4 & 6.61 & $\mathrm{~F}=6.83$, & $\mathrm{F}=20.5$, & $\mathrm{F}=25.8$, \\
& $(1.77)$ & $(1.87)$ & $(2.03)$ & $(1.12)$ & $\mathrm{p}<0.05$ & $\mathrm{p}<0.001$ & $\mathrm{p}<0.001$ \\
Con pareja & 4.36 & 3.97 & 4.06 & 6.64 & $\mathrm{~F}=12.9$, & $\mathrm{F}=19.0$, & $\mathrm{F}=25.6$, \\
& $(2.04)$ & $(1.89)$ & $(2.07)$ & $(1.04)$ & $\mathrm{p}<0.001$ & $\mathrm{p}<0.001$ & $\mathrm{p}<0.001$ \\
\hline
\end{tabular}


$\underline{\text { Tabla } 4 .}$

Comparación entre los grupos experimental y control sobre la comunicación y estilos de negociación con la pareja

\begin{tabular}{lcccccccc}
\hline \multirow{2}{*}{ Variable } & \multicolumn{2}{c}{ Control } & \multicolumn{2}{c}{ Experimental } & \multicolumn{3}{c}{ ANOVA de medidas repetidas } \\
& Pre & Post & Pre & Post & Grupo & Tratamiento & Interacción \\
\hline Comunicación sobre temas sexuales & 2.22 & 2.07 & 2.76 & 2.53 & $\mathrm{~F}=39.0$, & $\mathrm{F}=26.2$, & $\mathrm{F}=28.2$, \\
& $(1.27)$ & $(1.25)$ & $(1.46)$ & $(0.84)$ & $\mathrm{p}<0.001$ & $\mathrm{p}<0.001$ & $\mathrm{p}<0.001$ \\
$\begin{array}{l}\text { Estilos de negociación: colaboración- } \\
\text { equidad }\end{array}$ & 3.65 & 3.55 & 3.44 & 4.81 & $\mathrm{~F}=6.71$, & $\mathrm{F}=13.8$, & $\mathrm{F}=13.8$, \\
\hline
\end{tabular}

Al revisar los efectos principales del grupo respecto a la habilidad demostrada para usar correctamente un condón se encontró que existen diferencias significativas en el porcentaje de respuestas correctas en la habilidad para colocar un condón entre el grupo control y el experimental $(\mathrm{F}=247, p<0.001)$. El grupo experimental (98.61\%) obtuvo un mayor porcentaje que el control (18.01\%); y al analizar el efecto del tipo de tratamiento se observaron cambios en el porcentaje de respuestas correctas entre la medición pre $\mathrm{y}$ post $(\mathrm{F}=247, p<0.001)$. Por otra parte, en la interacción de estas variables se encontró que existe un cambio en el porcentaje de respuestas correctas entre pre y post diferenciado para cada grupo $(\mathrm{F}=390, p<0.001)$, pues se pudo observar que en el grupo control se incrementó un poco el porcentaje, mientras que el experimental incrementó de un $20 \%$ a un promedio cercano al $100 \%$ de aciertos.

En la habilidad para negociar el uso del condón también se realizó un análisis de medidas repetidas y al revisar los efectos principales de los grupos, se encontraron diferencias significativas en las puntuaciones obtenidas entre el grupo control y experimental $(\mathrm{F}=42.9, p<0.001)$; el grupo experimental obtuvo mayor habilidad para negociar el uso del condón que el grupo control; y al analizar el efecto del tipo de tratamiento se observaron cambios en la puntuación obtenida entre la medición pre y post $(\mathrm{F}=88.5, p<0.001)$. En la interacción se encontraron cambios en las puntuaciones entre los grupos ( $\mathrm{F}=88.5, p<0.001)$, pues el grupo control se mantuvo inhábil, mientras que en el grupo experimental hubo cambios en su habilidad de pre a post: más del $69 \%$ de los casos tuvo la habilidad para negociar el uso del condón en el grupo experimental, mientras que más del $60 \%$ del grupo control no pudo hacerlo.

\section{Variables del mesosistema}

En la comparación de medias entre el grupo experimental y el grupo control en las variables del mesosistema (véase Tabla 5), con respecto a las variables referidas a padre y madre, el grupo experimental obtuvo mayor percepción de apoyo y supervisión del padre y mayor frecuencia de la comunicación que el grupo control.

En el caso de las variables relacionadas con los amigos, los efectos principales del grupo muestran que el grupo experimental obtuvo mayores puntuaciones que el control en la norma subjetiva, así como en la autoeficacia para evitar conductas de riesgo de los amigos.

\section{Variables del macrosistema}

Al evaluar los estereotipos en el ámbito interpersonal y en el ámbito social se encontraron diferencias significativas en la puntuación obtenida entre los grupos: el grupo experimental obtuvo menores puntajes que el control en estereotipos en el ámbito interpersonal y social (véase Tabla 6). 
Tabla 5.

Comparación entre los grupos experimental y control sobre las variables del mesosistema

\begin{tabular}{|c|c|c|c|c|c|c|c|}
\hline \multirow{2}{*}{ Variable } & \multicolumn{2}{|c|}{ Control } & \multicolumn{2}{|c|}{ Experimental } & \multicolumn{3}{|c|}{ ANOVA de medidas repetidas } \\
\hline & Pre & Post & Pre & Post & Grupo & Tratamiento & Interacción \\
\hline \multicolumn{8}{|l|}{ Padre } \\
\hline Apoyo & $\begin{array}{c}2.60 \\
(1.56)\end{array}$ & $\begin{array}{c}2.86 \\
(1.68)\end{array}$ & $\begin{array}{c}3.14 \\
(1.78)\end{array}$ & $\begin{array}{l}4.69 \\
(.82)\end{array}$ & $\begin{array}{c}\mathrm{F}=18.0 \\
\mathrm{p}<0.001\end{array}$ & $\begin{array}{c}\mathrm{F}=25.4 \\
\mathrm{p}<0.001\end{array}$ & $\begin{array}{l}F=6.70 \\
p<0.05\end{array}$ \\
\hline Supervisión & $\begin{array}{c}2.69 \\
(1.58)\end{array}$ & $\begin{array}{c}2.66 \\
(1.71)\end{array}$ & $\begin{array}{c}3.05 \\
(1.76)\end{array}$ & $\begin{array}{l}4.67 \\
(.82)\end{array}$ & $\begin{array}{c}\mathrm{F}=16.9 \\
\mathrm{p}<0.001\end{array}$ & $\begin{array}{c}\mathrm{F}=23.9 \\
\mathrm{p}<0.001\end{array}$ & $\begin{array}{l}\mathrm{F}=11.5 \\
\mathrm{p}<0.001\end{array}$ \\
\hline Comunicación sobre temas sexuales & $\begin{array}{c}1.88 \\
(1.23)\end{array}$ & $\begin{array}{c}2.48 \\
(1.32)\end{array}$ & $\begin{array}{c}2.29 \\
(1.41)\end{array}$ & $\begin{array}{l}4.53 \\
(.84)\end{array}$ & $\begin{array}{c}F=21.8 \\
p<0.001\end{array}$ & $\begin{array}{c}\mathrm{F}=86.3 \\
\mathrm{p}<0.001\end{array}$ & $\begin{array}{l}\mathrm{F}=31.2 \\
\mathrm{p}<0.001\end{array}$ \\
\hline \multicolumn{8}{|l|}{ Madre } \\
\hline Apoyo & $\begin{array}{c}3.58 \\
(1.59)\end{array}$ & $\begin{array}{c}2.69 \\
(1.75)\end{array}$ & $\begin{array}{c}3.58 \\
(1.73)\end{array}$ & $\begin{array}{l}4.72 \\
(.81)\end{array}$ & $\begin{array}{l}F=13.6 \\
p<0.001\end{array}$ & $\begin{array}{l}F=.606 \\
p=0.439\end{array}$ & $\begin{array}{l}F=15.0 \\
p<0.001\end{array}$ \\
\hline Supervisión & $\begin{array}{c}3.44 \\
(1.66)\end{array}$ & $\begin{array}{c}2.69 \\
(1.81)\end{array}$ & $\begin{array}{c}3.55 \\
(1.74)\end{array}$ & $\begin{array}{l}4.69 \\
(.85)\end{array}$ & $\begin{array}{c}\mathrm{F}=14.0 \\
\mathrm{p}<0.001\end{array}$ & $\begin{array}{c}\mathrm{F}=.949 \\
\mathrm{p}=0.334\end{array}$ & $\begin{array}{l}\mathrm{F}=11.8 \\
\mathrm{p}<0.001\end{array}$ \\
\hline Comunicación sobre temas sexuales & $\begin{array}{c}2.02 \\
(1.25)\end{array}$ & $\begin{array}{c}2.31 \\
(1.36)\end{array}$ & $\begin{array}{c}2.11 \\
(1.27)\end{array}$ & $\begin{array}{c}4.47 \\
(1.00)\end{array}$ & $\begin{array}{c}\mathrm{F}=22.5 \\
\mathrm{p}<0.001\end{array}$ & $\begin{array}{c}\mathrm{F}=58.3 \\
\mathrm{p}<0.001\end{array}$ & $\begin{array}{l}\mathrm{F}=31.3 \\
\mathrm{p}<0.001\end{array}$ \\
\hline \multicolumn{8}{|l|}{ Amigos } \\
\hline \multicolumn{8}{|l|}{ Norma subjetiva: } \\
\hline Usar condón con pareja regular & $\begin{array}{c}4.34 \\
(2.78)\end{array}$ & $\begin{array}{c}4.07 \\
(2.68)\end{array}$ & $\begin{array}{c}4.90 \\
(2.62)\end{array}$ & $\begin{array}{c}6.44 \\
(1.64)\end{array}$ & $\begin{array}{l}F=7.04 \\
p<0.05\end{array}$ & $\begin{array}{l}F=5.65 \\
p<0.05\end{array}$ & $\begin{array}{l}F=7.80 \\
p<0.05\end{array}$ \\
\hline $\begin{array}{l}\text { Autoeficacia para evitar conductas de } \\
\text { riesgo }\end{array}$ & $\begin{array}{c}2.84 \\
(1.60)\end{array}$ & $\begin{array}{l}4.07 \\
(1.16)\end{array}$ & $\begin{array}{c}2.79 \\
(1.29)\end{array}$ & $\begin{array}{l}6.61 \\
(.87)\end{array}$ & $\begin{array}{l}F=36.8 \\
p<0.001\end{array}$ & $\begin{array}{l}F=107 \\
p<0.001\end{array}$ & $\begin{array}{l}F=31.6 \\
p<0.001\end{array}$ \\
\hline
\end{tabular}

Tabla 6.

Comparación entre los grupos experimental y control sobre las variables del mesosistema

\begin{tabular}{lccccccc}
\hline \multirow{2}{*}{ Variable } & \multicolumn{2}{c}{ Control } & \multicolumn{2}{c}{ Experimental } & \multicolumn{3}{c}{ ANOVA de medidas repetidas } \\
& Pre & Post & Pre & Post & Grupo & Tratamiento & Interacción \\
\hline \multirow{2}{*}{ Ámbito interpersonal } & 2.60 & 3.28 & 2.69 & 1.28 & $\mathrm{~F}=11.7$ & $\mathrm{~F}=3.93$ & $\mathrm{~F}=37.2$ \\
& $(1.40)$ & $(1.16)$ & $(1.44)$ & $(0.74)$ & $p<0.001$ & $p<0.05$ & $p<0.001$ \\
Ámbito social & 3.06 & 3.14 & 3.25 & 1.22 & $\mathrm{~F}=13.0$ & $\mathrm{~F}=25.3$ & $\mathrm{~F}=42.0$ \\
& $(1.39)$ & $(1.24)$ & $(1.22)$ & $(0.68)$ & $p<0.001$ & $p<0.001$ & $p<0.001$ \\
\hline
\end{tabular}

\section{DISCUSIÓN}

Los principales hallazgos de este estudio demuestran que el programa de intervención diseñado con base en el modelo ecológico de Bronfenbrenner (1987) tuvo un impacto positivo en el grupo experimental en todas las variables evaluadas, excepto en autoestima y en comunicación con pareja.

Respecto al nivel micro, cabe mencionar que en la preevaluación los adolescentes, de manera general, presentaron porcentajes muy bajos en conocimientos. Por esta razón, dotar a los jóvenes de información es el primer paso para lograr que identifiquen el riesgo de contraer el VIH, teniendo en cuenta que cuando las personas están informadas pueden adoptar una postura diferente (Amirkhanian, Kelly, Kabakchieva, McAuliffe y Vassileva, 2003).

Sobre las variables psicosociales, se encontró que antes de la intervención hubo diferencias significativas entre los grupos control y experimental en las variables que evalúan creencias hacia el uso del condón; sin embargo, cabe resaltar que para la postevaluación, a pesar de existir diferencias significativas, la interpretación es diferente, ya que el grupo experimental redujo las creencias negativas e incrementó las creencias de protección. Esto es relevante, ya que en la medida en que los adolescentes perciban que el condón puede protegerlos puede asociarse con un 
comportamiento sexual preventivo, así como lo demuestran diversas investigaciones (Gallegos, Villarruel, Loveland, Ronis y Zhou, 2008).

El programa de intervención también propició el incremento en las actitudes favorables hacia el uso del condón, tanto en la intención de uso como en sentirse más eficaces para usarlo. Posiblemente este hallazgo se deba, por un lado, al uso de la estrategia de anuncio publicitario que, como se ha visto en otros estudios, contribuye para que los adolescentes desarrollen una mejor actitud hacia el uso del condón (Callejas et al., 2005); y, por otro lado, al entrenamiento utilizado en habilidades para mejorar la eficacia en el uso del condón (Amirkhanian et al., 2003).

Por otro lado, respecto a los efectos de la intervención sobre la autoestima no se encontraron cambios en las medias obtenidas entre pre y postevaluación, pues en ambos casos los adolescentes reportaron tener una buena autoestima; probablemente esto se deba a que en el programa solo se hizo énfasis en esta variable en una sola sesión. Por lo tanto, para próximas intervenciones sería necesario trabajar este tema en un mayor número de sesiones y usar otras estrategias para mejorar la autoestima de los jóvenes, centradas en el empoderamiento y la toma de decisiones (Harper, Bangi, Sánchez, Doll y Pedraza, 2009).

Sobre la comunicación con padres y pareja, en la intervención se evaluó la autoeficacia para hablar con sus padres sobre algún tema sexual, por lo que al evaluar específicamente la percepción de los adolescentes, esta variable se ubicó en el nivel micro; los resultados obtenidos mostraron que al finalizar el programa, los adolescentes se mostraron más capaces de poder hacerlo. Asimismo, reportaron tener un estilo de negociación de colaboración-equidad sobre el uso del condón, lo cual puede suponer mayor éxito para usar condón en sus relaciones sexuales (Robles, Frias, Moreno, Rodriguez y Barroso, 2011). Por otro lado, a pesar de que los adolescentes reportaron tener un estilo de colaboraciónequidad cuando se realizó la preevaluación, no eran capaces de utilizar este estilo para negociar el uso del condón; por esto, cabe resaltar la importancia de evaluar no solo lo que ellos creen que hacen, sino también, a través de la observación directa, evaluar sus habilidades en la práctica.

En la habilidad para negociar el uso del condón se encontró que los adolescentes del grupo experimental mejoraron la habilidad y pudieron negociar su uso dando argumentos y debatiendo lo que la pareja les decía para no usarlo. Es importante resaltar que, dado que en el programa no fue posible evaluar la conducta sexual después de la intervención, en algunas investigaciones como las realizadas por Anderson et al. (2006) y Czuchry, Timpson, Williams, Bowen y Ratliff (2009), se encontró que mejorar las habilidades conductuales de negociación del uso del condón incrementa el uso de este en los participantes; y, siendo así, cuando en las actividades del programa se negocia el uso del condón y se obtiene lo deseado, teniendo información sobre las ventajas de su uso, los adolescentes se sienten más capaces de poderlo negociar en la práctica.

Con respecto a la habilidad para usar correctamente el condón, los resultados muestran diferencias significativas, después de la intervención, entre los grupos control y experimental. Aquellos que fueron entrenados en esta habilidad, así como se ha demostrado en investigaciones con otras poblaciones (Robles et al., 2006), tuvieron mayor porcentaje de respuestas correctas en la postevaluación. El desarrollo de esta habilidad es fundamental, dado que se ha encontrado que a pesar de que las personas se sientan autoeficaces para usar condón, esto no implica necesariamente que sepan usarlo de manera correcta; e incluso de aquellos que reportan usar el condón, no se sabe si lo usan de principio a fin durante el acto sexual, pues con el solo intercambio de fluidos genitales es suficiente para contagiarse de VIH. Cabe resaltar el hallazgo de que desarrollar correctamente la habilidad a través del entrenamiento con un pene para enseñanza disminuye la ocurrencia de fallas al usarlo en el acto sexual y, por tanto, se reduce el riesgo de infección por transmisión sexual (Robles et al., 2014).

Respecto a las variables que se encuentran en el mesosistema se encontró que el grupo experimental incrementó la percepción sobre el apoyo y la supervisión recibida por parte de sus padres y también fue mayor la frecuencia de comunicación sobre temas sexuales. Estos resultados deben ser tomados con reserva, ya que no se tuvo la oportunidad de una evaluación a los padres, y no se sabe con certeza qué tanto los cambios se debieron a la intervención, o a la deseabilidad social, es decir, a lo que se esperaba que los adolescentes respondieran en la postevaluación. Aunque estos cambios a favor harían suponer que se asocian a conductas preventivas (Andrade, Betancourt y Palacios, 2006), se sugiere incorporar a los padres dentro de los programas de intervención para poder evaluar efectivamente estos aspectos (Wang et al., 2007; Thurman et al., 2016).

En torno a la influencia de los amigos sobre el comportamiento sexual, se intervino sobre la norma subjetiva para usar condón; es decir, se trató de que percibieran que para sus amigos es importante el uso del condón, e incluso dentro del mismo grupo de pares en la comunidad de diagnóstico. Esto cobra importancia porque cuando los adolescentes perciben que para sus amigos es importante el uso del condón, es más probable que lo usen (Anderson et al., 2006), y es mayor la intención de usarlo en la próxima relación sexual (Gallegos et al., 2008)

Con respecto al nivel macro se intervino sobre estereotipos de género. Los adolescentes que se encontraban en el 
grupo experimental redujeron sus creencias estereotipadas sobre hombres y mujeres, y fue menor el porcentaje de adolescentes que creyeron que el hombre es superior a la mujer. En los programas de intervención es necesario trabajar sobre el rol de género y las relaciones de poder que se establecen en la pareja para promover el uso del condón (Czuchry et al., 2009); y, de acuerdo con Centeno (2008), también se deben crear estrategias encaminadas a reducir el estigma y la discriminación de las personas infectadas, dado que hay una estrecha relación con el comportamiento sexual. Este último punto no fue considerado dentro del programa de intervención, por lo que se recomienda incluirlo en futuras intervenciones.

Como se puede observar, en general se encontraron diferencias significativas a favor del grupo experimental, en comparación con el grupo control; y cabe mencionar que los cambios deben considerarse en conjunto dado que el modelo propone diferentes niveles para explicar el comportamiento sexual (DiClemente et al., 2007); por ejemplo, incrementar el nivel de información y desarrollar las habilidades para negociar el uso del condón y de cómo usarlo de manera correcta pudo haber favorecido el cambio en la habilidad para negociar el condón con la pareja, ya que le da elementos al adolescente para que pueda negociar su uso. De igual forma, el entrenamiento en habilidades puede garantizar que se sientan más capaces de poder usar el condón.

Algo importante es que, aunque no se intervino directamente con los padres, se solicitó que como tarea hablaran sobre algún tema de sexualidad con ellos, lo que puede sugerir que los padres se involucren y hablen más con sus hijos sobre salud sexual. De igual forma, el disminuir los estereotipos de género contribuye a que cambien sus creencias sobre lo que hace un hombre y una mujer en el terreno sexual y puedan incrementar conductas preventivas, dado que se responsabilizan de qué hacer cuando se tienen relaciones sexuales.

Dentro de las limitaciones de la investigación, se señala que no fue posible evaluar los efectos de la intervención sobre la conducta sexual; por tanto, se sugiere hacer un seguimiento a los adolescentes que hayan egresado de la comunidad, y evaluar el impacto del programa en su conducta sexual. Por otro lado, fue difícil poder tener todas las evaluaciones de los jóvenes debido a su fluctuación y estadía en la comunidad, por lo que es necesario crear intervenciones breves que permitan una menor mortalidad experimental. Finalmente, se trabajó únicamente con los adolescentes, pero es necesario intervenir también con los padres dada la importancia que tienen en el desarrollo de sus hijos.

A modo de conclusión, es necesario hacer intervenciones breves, por un lado, para poder evaluar los efectos de la intervención en todos los usuarios; por otro, para capacitar al personal de la comunidad para que sean ellos quienes trabajen constantemente en la prevención del VIH con la población (O’Laughlin, 2015); y también, considerar los contextos que se asocien con la conducta sexual de los jóvenes en conflicto con la ley para tener intervenciones eficaces y con efectos a largo plazo. Asimismo, se requiere seguir replicando este programa de intervención para evaluar su efectividad, y adicionalmente, evaluar a los adolescentes una vez que egresen de la comunidad para analizar su conducta sexual. Y finalmente, se concluye que esta investigación muestra evidencia empírica de la efectividad de programas de intervención diseñados a partir del modelo ecológico para promover la salud sexual.

\section{REFERENCIAS}

Amirkhanian, Y., Kelly, J., Kabakchieva, E., McAuliffe, T. y Vassileva, S. (2003). Evaluation of a social network HIV prevention intervention program for young men who have sex with men in Russia and Bulgaria. AIDS Education and Prevention, 15(3), 205-215.

Anderson, E., Wagstaff, D., Heckman, T., Winett, R., Roffman, R., Solomon, L. et al. (2006). Information-motivation-behavioral skills (IMB) model: Testing direct and mediated treatment effects on condom use among women in low-income housing. Annals of Behavioral Medicine: A Publication of the Society of Behavioral Medicine, 31(1), 70-79.

Andrade, J., Barbosa, J. y Lozada, C. (2012). Factores de riesgo biopsicosocial que influyen en el desarrollo del trastorno disocial en adolescentes colombianos. Revista Internacional de Psicología, 12(1), 1-25.

Andrade, P., Betancourt, D. y Palacios, J. (2006). Factores familiares asociados a la conducta sexual en adolescentes. Revista Colombiana de Psicología, 15, 91-101.

Bronfenbrenner, U. (1987). La ecología del desarrollo humano. Experimentos en entornos naturales y diseñados. Barcelona: Paidós.

Callejas, S., Fernández, B., Méndez, P., León, T., Fábrega, C., Villarín, A. et al. (2005). Intervención educativa para la prevención de embarazos y enfermedades de transmisión sexual en adolescentes de la Ciudad de Toledo. Revista Española de Salud Pública, 79(5), 581-589.

Centeno, H. (2008). Fortalezas y debilidades del proyecto de atención a poblaciones móviles para la disminución del VIH/ SIDA en El Salvador, una intervención de campo. Población y Salud en Mesoamérica, 5(2), 1-16.

Czuchry, M., Timpson, S., Williams, M., Bowen, A. M. y Ratliff, E. A. (2009). Improving condom self-efficacy and use among individuals living with HIV: the positive choices mapping intervention. Journal of Substance Use, 14(3-4), 230-239.

DGTPA. (2013). Sitio web de la Dirección General de Tratamiento para Adolescentes. Recuperado de http://www.reclusorios. cdmx.gob.mx/adolescentes/index.html 
Díaz, E. (2010). Relación de la autoestima con el comportamiento sexual de los adolescentes. Tesis de Especialidad no publicada. UniverSIDAd Nacional Autónoma de México, Morelia, Michoacan.

DiClemente, R., Salazar, L. y Crosby, R. (2007). A review of STD/HIV preventive interventions for adolescents: Sustaining effects using an ecological approach. Journal of Pediatric Psychology, nil(nil), 1-19.

Gallegos, E., Villarruel, A., Loveland, C., Ronis, D. y Zhou, Y. (2008). Intervención para reducir riesgo en conductas sexuales de adolescentes: Un ensayo aleatorizado y controlado. Salud Pública de México, 50(1), 59-66.

Gaxiola, J., González, S. y Contreras, Z. (2011). Resiliencia, metas y contexto social: su influencia en el rendimiento académico en estudiantes de preparatoria. Trabajo presentado en el XXXVIII Congreso Nacional del Consejo Nacional para la Enseñanza e Investigación en Psicología (CNEIP). Ciudad de México, México.

Harper, G. W., Bangi, A. K., Sánchez, B., Doll, M. y Pedraza, A. (2009). A quasi-experimental evaluation of a communitybased HIV prevention intervention for Mexican American female adolescents: the SHERO's program. AIDS Education y Prevention, 21(5), 109-123.

Kerlinger, F. y Lee, H. (2002). Investigación del comportamiento. Métodos de investigación en ciencias sociales (4a. ed.). México: McGraw Hill.

Ministerio de la Protección Social de Colombia. (2011). Guía de Prevención VIH/SIDA. Población privada de la libertad. Recuperado de https://www.minsalud.gov.co/salud/Documents/ observatorio_vih/documentos/prevencion/promocion_prevencion/prevencion_poblaciones_vulnerables/a_poblaciones_vulnerables/PPL.pdf

O'Laughlin, B. (2015). Trapped in the prison of the proximate: structural HIV/AIDS prevention in southern Africa. Review of African Political Economy, 42(145), 342-361.

ONUSIDA. (2009). Las mujeres y el VIH en el entorno carcelario. Recuperado de https://www.unodc.org/documents/hiv-aids/ UNODC_UNAIDS_2008_Women_and_HIV_in_prison_settings-SP.pdf

ONUSIDA. (2014). The GAP Report. Recuperado de: http://www. unaids.org/sites/default/files/en/media/unaids/contentassets/ documents/unaidspublication/2014/UNAIDS_Gap_report_en.pdf

ONUSIDA. (2016). Fast-Track to ending AIDS. Recuperado de: http://www.unaids.org/sites/default/files/media_ asset/2016HighLevelMeeting_en.pdf

Operario, D., Smith, C. D., Arnold, E. y Kegeles, S. (2010). The Bruthas Project: evaluation of a community-based HIV prevention intervention for African American men who have sex with men and women. AIDS Education y Prevention, 22(1), 37-48.

Pinto, R. M. y McKay, M. M. (2006). A mixed-method analysis of African-American women's attendance at an HIV preven- tion intervention. Journal of Community Psychology, 34(5), 601-616.

Robles, S. y Díaz-Loving, R. (2011). Validación de la Encuesta Estudiantil sobre Salud Sexual (EESS). México: Facultad de Estudios Superiores Iztacala.

Robles, S., Frias, B., Moreno, D., Rodriguez, M. y Barroso R. (2011). Conocimientos sobre VIH/SIDA, comunicación sexual y negociación del uso del condón en adolescentes sexualmente activos. Revista Electronica de Psicología Iztacala, 14(4), 317-333.

Robles, S., Moreno, D., Frías, B., Rodríguez, M., Barroso, R., Díaz-González, E. et al. (2006). Entrenamiento conductual en habilidades de comunicación sexual en la pareja y uso correcto del condón. Anales de Psicología, 22(1), 60-71.

Robles, S., Rodríguez, M., Frías, B. y Moreno, D. (2014). Indicadores del uso eficaz del preservativo. Revista Puertorriqueña de Psicología, 25(2), 244-258.

Rocha, T. y Díaz-Loving, R. (2005). Cultura de Género: la brecha ideológica entre hombres y mujeres. Anales de Psicología, 21(1), 42-49.

Sanabria, A. y Uribe, A. (2010). Factores psicosociales de riesgo asociados a conductas problemáticas en jóvenes infractores y no infractores. Diversitas: Perspectivas en Psicología, 6(2), 257-274.

Sánchez, R., Enríquez, D. y Reyes-Lagunes, I. (2011). Apoyo y supervisión parental y conducta sexual protegida. En DíazLoving, R., y Robles, S. (Eds.), Salud y sexualidad. México: Facultad de Estudios Superiores Iztacala.

Senteio, C., Wright, S., Jackson, R., Welk, S. y Zhang, S. (2010). Effective resources supporting healthy sexual behavior in formerly incarcerated persons. American Journal of Sexuality Education, 5, 362-376.

Thurman, T., Kidman, R., Carton, T. y Chiroro, P. (2016). Psychological and behavioral interventions to reduce HIV risk: evidence from a randomized control trial among orphaned and vulnerable adolescents in South Africa, AIDS Care, 28(1), 8-15.

Trickett, E. (2009). Multilevel community-based culturally situated interventions and community impact: An ecological perspective. Am J Community Psychol, 43, 257-266.

Tulloch, H., McCaul, K., Miltenberger, R. y Smyth, J. (2004). Partner communication skills and condom use among college couples. Journal of American College Health, 52(6), 263-267.

Wang, B., Li, X., Stanton, B., Kamali, V., Naar-King, S., Shah, I. et al. (2007). Sexual attitudes, pattern of communication, and sexual behavior among unmarried out-of-school youth in China. BMC Public Health, 7, 1-10.

Zimmerman, R., Cupp, P., Donohew, L., Sionéan, K., Feist-Price, S., y Helme, D. (2008). Effects of a School-Based, TheoryDriven HIV And Pregnancy Prevention Curriculum. Perspectives on Sexual and Reproductive Health, 40(1), 42-52. 